\title{
ASYMPTOTICS FOR THE FIRST PASSAGE TIMES OF LÉVY PROCESSES AND RANDOM WALKS
}

\author{
DENIS DENISOV, ${ }^{*}$ Cardiff University \\ VSEVOLOD SHNEER, ${ }^{* *}$ Heriot-Watt University
}

\begin{abstract}
We study the exact asymptotics for the distribution of the first time, $\tau_{x}$, a Lévy process $X_{t}$ crosses a fixed negative level $-x$. We prove that $\mathbb{P}\left\{\tau_{x}>t\right\} \sim V(x) \mathbb{P}\left\{X_{t} \geq 0\right\} / t$ as $t \rightarrow \infty$ for a certain function $V(x)$. Using known results for the large deviations of random walks, we obtain asymptotics for $\mathbb{P}\left\{\tau_{x}>t\right\}$ explicitly in both light- and heavy-tailed cases.
\end{abstract}

Keywords: Lévy process; random walk; busy period; first passage time; subexponential distribution; large deviation; single-server queue

2010 Mathematics Subject Classification: Primary 60G50; 60G51

Secondary $60 \mathrm{~K} 25$

\section{Introduction}

Let $\left\{X_{t}\right\}_{t \geq 0}$ be a Lévy process with the characteristic function $\mathbb{E}\left\{\mathrm{e}^{i \theta X_{t}}\right\}=\mathrm{e}^{t \Psi(\theta)}$ for all $t \geq 0$, where $\Psi$ is given by the Lévy-Khinchine formula [29]

$$
\Psi(\theta)=\mathrm{i} A \theta-\frac{1}{2} \sigma^{2} \theta^{2}+\int_{-\infty}^{+\infty}\left(\mathrm{e}^{\mathrm{i} \theta x}-1-\mathrm{i} \theta x \mathbf{1}_{[-1,1]}\right) \Pi(\mathrm{d} x) .
$$

For $x \geq 0$, let

$$
\tau_{x}=\min \left\{t \geq 0: X_{t}<-x\right\}
$$

be the first passage time. Throughout, we assume that the Lévy process $X_{t}$ drifts to $-\infty$ almost surely (a.s.). Due to Rogozin's criterion [27] (see also [4, p. 167] or [29, Theorem 48.1]), $X_{t} \rightarrow-\infty$ if and only if

$$
\int_{1}^{\infty} t^{-1} \mathbb{P}\left\{X_{t} \geq 0\right\} \mathrm{d} t<\infty .
$$

This assumption implies that $\tau_{x}$ is a proper random variable with a finite expectation:

$$
\tau_{x}<\infty \quad \text { a.s., } \quad \mathbb{E}\left\{\tau_{x}\right\}<\infty \text {. }
$$

The aim of this paper is to study the asymptotical behaviour of

$$
\mathbb{P}\left\{\tau_{x}>t\right\}
$$

when $x>0$ is fixed and $t \rightarrow \infty$.

Received 16 February 2009; revision received 15 August 2012.

* Postal address: School of Mathematics, Cardiff University, Senghennydd Road, Cardiff, CF24 4AG, UK.

** Postal address: Department of AMS, Heriot-Watt University, Edinburgh, EH14 4AS, UK.

Email address: v.shneer@hw.ac.uk 
A similar problem may be addressed in discrete time, where an analogue of Lévy processes is random walks. If we denote by $S_{n}=\xi_{1}+\cdots+\xi_{n}$ a random walk with independent and identically distributed (i.i.d.) increments and assume that $S_{n} \rightarrow-\infty$ a.s., then an analogue of $\tau_{x}$ is the stopping time $v_{x}=\min \left\{n \geq 1: S_{n}<-x\right\}$. Asymptotics for the tail of $v_{x}$ were studied by various authors in many cases. In [15] these asymptotics were found for $x=0$; it was shown that $\mathbb{P}\left\{v_{0}>n\right\} \sim \mathbb{P}\left\{S_{n} \geq 0\right\} / n$ as $n \rightarrow \infty$ if the right-hand side is a subexponential sequence (for the definition, see Section 2). In [5] and [13] the asymptotics for the tail of $v_{x}$ have been found when $x>0$. In these papers the authors considered separately several classes of distribution of $\xi$ : regularly varying distributions, distributions satisfying Cramér's condition, and light-tailed distributions that do not satisfy Cramér's condition. For each of these classes, they showed that $\mathbb{P}\left\{v_{x}>n\right\}$ is asymptotically proportional to $\mathbb{P}\left\{S_{n} \geq 0\right\} / n$ using large deviation asymptotics for $\mathbb{P}\left\{S_{n} \geq 0\right\}$. In [9] (see also [8]) it was shown that the same asymptotic equivalence holds for the so-called semiexponential distributions with parameter smaller that $\frac{1}{2}$ (see the definition in [8]). In [22] the latter result has been generalised to the case of semiexponential distributions with parameter smaller than 1. We also note that in [22] $x$ may depend on $n$. We also mention the paper [7] where the same problem was studied under Cramér's assumptions and in the case when $x$ may depend on $n$.

For Lévy processes, the asymptotics for $\tau_{x}$ have not received much attention so far. We are aware of only the paper [21], where these asymptotics were obtained for the light-tailed distributions. However, necessary and sufficient conditions for the existence of moments of $\tau_{x}$ were obtained in [14].

In this paper we extend the approach proposed in [5] and [13]. Our main results are Theorems 2.1 and 2.2. Theorem 2.1 states that, under some natural assumptions,

$$
\mathbb{P}\left\{\tau_{x}>t\right\} \sim V(x) \frac{\mathbb{P}\left\{X_{t} \geq 0\right\}}{t}
$$

as $t \rightarrow \infty$ for a function $V(x)$ depending only on $x$. Theorem 2.2 shows that, under identical conditions for both Lévy processes and random walks,

$$
\mathbb{P}\left\{v_{x}>n\right\} \sim V_{r w}(x) \frac{\mathbb{P}\left\{S_{n} \geq 0\right\}}{n}, \quad \mathbb{P}\left\{\tau_{x}>t\right\} \sim V(x) \mathrm{e}^{-\gamma(t-[t])} \frac{\mathbb{P}\left\{X_{[t]} \geq 0\right\}}{t}
$$

for some $\gamma \geq 0$, where by $[t]$ we denote the integer part of $t$ (the largest integer smaller than $t$ ), and the functions $V(x)$ and $V_{r w}(x)$ depend only on $x$.

We would like to point out that, for the case of Lévy processes, both conditions of Theorem 2.2 and its result are given in terms of the values of the process at discrete times. Therefore, the problem of finding asymptotics for $\mathbb{P}\left\{\tau_{x}>t\right\}$ and $\mathbb{P}\left\{v_{x}>n\right\}$ is reduced to finding asymptotics for $\mathbb{P}\left\{S_{n} \geq 0\right\}$, or $\mathbb{P}\left\{\tilde{S}_{n} \geq n a\right\}$, where $a=-\mathbb{E}\left\{\xi_{1}\right\}$ and $\tilde{S}_{n}=S_{n}+n a$ is a random walk with zero drift. This is a problem of large deviations of sums of i.i.d. random variables which is extensively studied in the literature. We apply known results to obtain explicit asymptotics in various cases. It appears that in all cases when asymptotics for $\mathbb{P}\left\{S_{n}>0\right\}$ can be found explicitly, the conditions of Theorem 2.2 are satisfied and, hence, asymptotics for the tail distribution of $\tau_{x}$ and $v_{x}$ can also be found explicitly.

We would like to note that our main results provide a unified approach for finding asymptotics of $\tau_{x}$ and $v_{x}$. We do not make specific assumptions on the distribution of the increments of a Lévy process or a random walk.

After formulating our main results, we give corollaries for specific distributions. We consider distributions with heavy tails (such that $\mathbb{E}\left\{\mathrm{e}^{\varepsilon \xi_{1}}\right\}=\infty$ for all $\varepsilon>0$ ) and distributions with light 
tails (for which the latter condition is not fulfilled) separately. It was pointed out by various authors that, for the problem of large deviations of sums of heavy-tailed random variables, one should indicate two classes of distributions: those with tails lighter and heavier than $\mathrm{e}^{-\sqrt{t}}$ (we say that the tail of a distribution $F$ is lighter than a function $f$ if $\bar{F}(t) / f(t) \rightarrow 0$ as $t \rightarrow \infty$ and heavier than a function $f$ if $\bar{F}(t) / f(t) \rightarrow \infty$ ). Not surprisingly, the boundary $\mathrm{e}^{-\sqrt{t}}$ arises in our investigation as well.

Theorem 2.2 can also be applied to the case of light-tailed distributions under some further assumptions. In particular, the conditions of Theorem 2.2 are fulfilled if the distribution of $\xi_{1}$ satisfies the so-called Cramér's (or classical) conditions. With the help of Theorem 2.2 we can also cover the so-called intermediate case, when $\xi_{1}$ has a distribution with a light tail but does not satisfy Cramér's condition.

Another motivation for our work was to find asymptotics for the busy period in a stable M/G/1 queue. Let $A_{1}, A_{2}, \ldots$ and $B_{1}, B_{2}, \ldots$ be two mutually independent sequences, each consisting of i.i.d. random variables. Assume that the $\left\{A_{i}\right\}$ are interarrival times and that the $\left\{B_{i}\right\}$ are service times. We assume throughout that $\mathbb{E}\left\{B_{1}\right\} / \mathbb{E}\left\{A_{1}\right\}=\rho<1$, so that the system is stable. We use standard notation: an $\mathrm{M} / \mathrm{G} / 1$ system consists of $A_{i}$ exponential random variables and a GI/GI/1 system consists of a general i.i.d. sequence $\left\{A_{i}\right\}$. Define $N(t)=\max \left\{n: A_{1}+\cdots+A_{n} \leq t\right\}$. Put $X_{0}=0$ and

$$
X_{t}=\sum_{i=1}^{N(t)} B_{i}-t
$$

Then the busy period of the system with initial work $x>0$ may be defined as

$$
b p(x)=\inf \left\{t: X_{t}<-x\right\} .
$$

Hence, in an $\mathrm{M} / \mathrm{G} / 1$ queue finding asymptotics for the tail of $b p(x)$ is equivalent to finding asymptotics for the tail of $\tau_{x}$ when $X_{t}$ is a compound Poisson process without negative jumps, and, thus, our results may be applied.

The tail behaviour of the busy period in these systems has been a subject of interest for many researchers. Under Cramér-type assumptions, asymptotics for the $\mathrm{M} / \mathrm{G} / 1$ and $\mathrm{GI} / \mathrm{G} / 1$ settings were respectively studied in [1] and [24]. Most of the papers on the tail behaviour of the busy period are devoted to studying the case when $B_{1}$ has a subexponential distribution. All these papers investigate the asymptotic behaviour of $b p-$ a generic busy period, or a busy period of the queue under the condition that the first customer arriving at the system finds it empty. In [11], it was shown that if $B_{1}$ has a regularly varying distribution then

$$
\mathbb{P}\{b p>t\} \sim \mathbb{E}\left\{v_{0}\right\} \mathbb{P}\left\{B_{1}>(1-\rho) t\right\}
$$

as $t \rightarrow \infty$. This result was generalised in [30] to the case of a GI/GI/1 queue and under the assumption that the tail $\bar{B}(t)=\mathbb{P}\left\{B_{1}>t\right\}$ satisfies an extended regular variation condition (see [6]).

Later on, it was shown in [3] and [18] that the asymptotics in (1.3) hold for the GI/GI/1 model in the case when $B_{1}$ belongs to another subclass of heavy-tailed distributions which includes the Weibull distributions with parameter $\alpha<\frac{1}{2}$. The tails of the distributions considered in [3] and [18] are heavier than $\mathrm{e}^{-\sqrt{t}}$. As is shown in [2] (see also [17]), the latter condition is crucial for the asymptotics in (1.3) to hold.

The results of Theorem 2.2 allow us to find asymptotics for $\mathbb{P}\{b p(x)>t\}$ in the $\mathrm{M} / \mathrm{G} / 1$ queue for both light- and heavy-tailed distributions. Moreover, we are able to obtain these asymptotics when $\mathbb{P}\{B>t\}$ is lighter than $\mathrm{e}^{-\sqrt{t}}$ but still heavier than any exponential distribution, and in 
this case no results have been known until now. Using the results on the tail asymptotics of the distribution of $b p(x)$, we can also obtain results for the tail asymptotics of the distribution of $b p$.

We would like to underline the main contributions of this paper once again. Firstly, Theorem 2.2 provides a unified approach to finding asymptotics for the tails of $\tau_{x}$ and $v_{x}$ under rather general assumptions on the distribution of the increments. The conditions as well as the results of this theorem even for the Lévy processes are formulated in terms of the behaviour of $\mathbb{P}\left\{S_{n}>0\right\}$, which is a well-studied large deviations problem. Secondly, using some known results on large deviations, we find explicit asymptotics for the tails of $\tau_{x}$ and $v_{x}$, all of which are new in the case of $\tau_{n}$ and some of which are new in the case of $v_{x}$. Thirdly, our results could be applied to finding asymptotics for the tail distribution of a busy period of an $\mathrm{M} / \mathrm{G} / 1$ queue - a very important problem in queueing theory — and, in particular, we present new results concerning these asymptotics in the case when the service time distribution has a heavy tail which is lighter than $\mathrm{e}^{-\sqrt{t}}$.

The rest of the paper is organised as follows. In Section 2 we present Theorems 2.1 and 2.2 that reduce the problem of finding asymptotics for $\mathbb{P}\left\{\tau_{x}>t\right\}$ and $\mathbb{P}\left\{v_{x}>n\right\}$ to studying the asymptotics of $\mathbb{P}\left\{S_{n} \geq 0\right\}$. In Section 3 we consider four classes of distributions: heavytailed distributions I (with tails heavier than $\mathrm{e}^{-\sqrt{t}}$ ), heavy-tailed distributions II (with tails lighter than $\mathrm{e}^{-\sqrt{t}}$ ), distributions satisfying Cramér's condition, and distributions forming an intermediate case (distributions with light tails not satisfying Cramér's condition). For each of these cases, we give known results on the asymptotics of $\mathbb{P}\left\{S_{n}>0\right\}$, show that the conditions of Theorem 2.2 are satisfied, and derive results on the tail asymptotics of the distributions of $\tau_{x}$ and $v_{x}$. Appendix A is devoted to the proofs of Theorems 2.1 and 2.2, and in Appendix B we present some known results on Lévy processes that are used in our paper.

\section{Main results}

In this section we present Theorems 2.1 and 2.2 which connect the asymptotics for $\tau_{x}$ and $v_{x}$ with the asymptotics for $\mathbb{P}\left\{S_{n} \geq 0\right\}$. We start with some preliminaries.

Definition 2.1. A function $f: \mathbb{R}^{+} \rightarrow \mathbb{R}^{+}$belongs to the class $\delta d(\gamma)$ with $\gamma \geq 0$ if $f(t)>0$ for all large enough $t$, and

$$
\begin{gathered}
\lim _{t \rightarrow \infty} \frac{f(t-y)}{f(t)}=\mathrm{e}^{\gamma y} \quad \text { for any } y \in \mathbb{R} \\
\lim _{t \rightarrow \infty} \frac{f^{* 2}(t)}{f(t)}=\lim _{t \rightarrow \infty} \frac{\int_{0}^{t} f(t-y) f(y) \mathrm{d} y}{f(t)}=2 d=2 \int_{0}^{\infty} \mathrm{e}^{\gamma y} f(y) \mathrm{d} y .
\end{gathered}
$$

The class $\delta d:=\varsigma d(0)$ is called the class of subexponential densities.

A discrete-time analogue of this definition is as follows.

Definition 2.2. A sequence $\left\{a_{n}\right\}_{n \geq 0}$ belongs to the class $s_{s}(\gamma)$ with $\gamma \geq 0$ if $a_{n}>0$ for all large enough $n$, and

$$
\begin{gathered}
\lim _{n \rightarrow \infty} \frac{a_{n-1}}{a_{n}}=\mathrm{e}^{\gamma}, \\
\lim _{n \rightarrow \infty} \frac{a_{n}^{* 2}}{a_{n}} \equiv \lim _{n \rightarrow \infty} \frac{\sum_{i=0}^{n} a_{i} a_{n-i}}{a_{n}}=2 d=2 \sum_{i=0}^{\infty} a_{i} \mathrm{e}^{\gamma i} .
\end{gathered}
$$

The class $\ell_{s}:=\varsigma_{s}(0)$ is called the class of subexponential sequences. 
Theorem 2.1. Let the function

$$
\frac{\mathbb{P}\left\{X_{t} \geq 0\right\}}{t}, \quad t \geq 1,
$$

belong to the class $\delta d(\gamma)$. In addition, assume that there exists an $\alpha \geq 0$ such that

$$
\lim _{t \rightarrow \infty} \frac{\mathbb{P}\left\{X_{t} \geq 0\right\}}{\mathbb{P}\left\{X_{t} \geq y\right\}}=\mathrm{e}^{\alpha y} \quad \text { for any fixed } y .
$$

Then

$$
\mathbb{P}\left\{\tau_{x}>t\right\} \sim V(x) \frac{\mathbb{P}\left\{X_{t} \geq 0\right\}}{t}
$$

as $t \rightarrow \infty$ for any $x$ being a point of continuity of the function

$$
V(x) \equiv \begin{cases}\mathbb{E}\left\{\tau_{x}\right\}, & \gamma=\alpha=0, \\ \mathrm{e}^{\alpha x} \int_{0}^{\infty} \mathrm{e}^{\gamma t} \mathbb{E}\left\{\mathrm{e}^{\alpha N_{t}} ;\left|N_{t}\right| \leq x\right\} \mathrm{d} t, & \text { otherwise, }\end{cases}
$$

where $N_{t}=\inf _{0 \leq s \leq t} X_{s}$.

Note that the second formula in (2.5) reduces to $\mathbb{E}\left\{\tau_{x}\right\}$ in the case $\alpha=\gamma=0$.

In the next theorem we deal with both random walks and Lévy processes under identical assumptions.

Theorem 2.2. Assume that either a Lévy process $X_{t}$ or a random walk $X_{n}$ is given. Let the sequence

$$
\frac{\mathbb{P}\left\{X_{n} \geq 0\right\}}{n}, \quad n \in \mathbb{N},
$$

belong to the class $\wp_{s}(\gamma)$. In addition, assume that there exists an $\alpha \geq 0$ such that

$$
\lim _{n \rightarrow \infty} \frac{\mathbb{P}\left\{X_{n} \geq 0\right\}}{\mathbb{P}\left\{X_{n} \geq y\right\}}=\mathrm{e}^{\alpha y} \quad \text { for any fixed } y, n \in \mathbb{N} .
$$

Then, for a Lévy process $X_{t}$,

$$
\mathbb{P}\left\{\tau_{x}>t\right\} \sim V(x) \mathrm{e}^{-\gamma(t-[t])} \frac{\mathbb{P}\left\{X_{[t]} \geq 0\right\}}{t}
$$

as $t \rightarrow \infty$ for any $x$, where $V(x)$ is defined in Theorem 2.1.

For a random walk $X_{n}$,

$$
\mathbb{P}\left\{v_{x}>n\right\} \sim V_{r w}(x) \frac{\mathbb{P}\left\{X_{n} \geq 0\right\}}{n}
$$

as $n \rightarrow \infty$, where

$$
V_{r w}(x)= \begin{cases}\mathbb{E} v_{x}, & \gamma=\alpha=0, \\ \mathrm{e}^{\alpha x} \sum_{k=0}^{\infty} \mathrm{e}^{\gamma k} \mathbb{E}\left\{\mathrm{e}^{\alpha N_{k}} ;\left|N_{k}\right| \leq x\right\}, & \text { otherwise, }\end{cases}
$$

with $N_{k}=\min _{0 \leq l \leq k} X_{l}$.

Result 2.1. The conditions of Theorem 2.1 and (or) the conditions of Theorem 2.2 imply that $\mathrm{e}^{-\gamma}=\mathbb{E}\left\{\mathrm{e}^{\alpha X_{1}}\right\}$. The proof of this fact is given in Appendix A. Note also that this fact implies that $\alpha=0$ if and only if $\gamma=0$. This corresponds to the subexponential case. 


\section{Explicit results}

In the previous section we presented results that link the tail asymptotics of $\tau_{x}$ and $v_{x}$ with the large deviation probabilities for sums of i.i.d. random variables. The results of the previous section are, however, not explicit as they are given in terms of the large deviation probabilities and as the knowledge of more large deviation probabilities is also required to check the conditions of Theorem 2.2. This section is devoted to the derivation of explicit asymptotics for a (very) large number of distributions. It consists of four subsections. All these subsections have a similar structure: we define a class of distributions for which we present known results on large deviations of sums of random variables. After that, with the help of these results, we show that the conditions of Theorem 2.2 are satisfied and, as a result, we obtain the asymptotics for the tail distributions of $\tau_{x}$ and $v_{x}$.

First, we prove Theorem 3.2 in which we study the case when $-\ln \mathbb{P}\left\{X_{1}>t\right\}=o(\sqrt{t})$ (corresponding to the distributions with tails heavier than $\mathrm{e}^{-\sqrt{t}}$ ). Furthermore, in Theorem 3.4, we analyse the case when $-\ln \mathbb{P}\left\{X_{1}>t\right\}$ is regularly varying with parameter $\alpha \in\left[\frac{1}{2}, 1\right)$ (corresponding to the distributions with tails that are heavy but lighter than $\mathrm{e}^{-\sqrt{t}}$ ). Then, in Theorem 3.5 we give the asymptotics for Cramér's case. This includes (partially) the distributions with exponential tails and tails that are lighter than exponential. Finally, in Theorem 3.7 we analyse distributions with exponential tails that are not covered by Cramér's case. As corollaries, we give corresponding results for the tail asymptotics of the busy period of an $\mathrm{M} / \mathrm{G} / 1$ queue.

\subsection{Heavy-tailed distributions I}

The result below follows directly from Corollary 2.1 of [12].

Theorem 3.1. Let $S_{n}=\sum_{i=1}^{n} \xi_{i}$ be a random walk. Let $\mathbb{E}\left\{\xi_{1}\right\}=0$ and $\mathbb{E}\left\{\left|\xi_{1}\right|^{\kappa}\right\}<\infty$ for some $\kappa \in(1,2]$. Assume that

$$
\frac{\bar{F}\left(n-n^{1 / \kappa}\right)}{\bar{F}(n)} \rightarrow 1
$$

as $n \rightarrow \infty$, and also assume that

$$
\varepsilon(n) \equiv \sup _{x \geq 2 n^{1 / \kappa}} \frac{\mathbb{P}\left\{\xi_{1}>n^{1 / \kappa}, \xi_{2}>n^{1 / \kappa}, S_{2}>x\right\}}{\bar{F}(x)}=o\left(\frac{1}{n}\right)
$$

as $n \rightarrow \infty$. Then

$$
\mathbb{P}\left\{S_{n}>n a\right\} \sim n \mathbb{P}\left\{\xi_{1}>n a\right\}
$$

as $n \rightarrow \infty$ for any $a>0$.

For the tail asymptotics of $\tau_{x}$ and $v_{x}$ the following is true.

Theorem 3.2. Assume that either a Lévy process $X_{t}$ or a random walk $X_{n}$ is given. Let $\mathbb{E}\left\{X_{1}\right\}=-a<0$, and assume that the distribution of the random variable $X_{1}+$ a satisfies the conditions of Theorem 3.1. Then

$$
\begin{gathered}
\mathbb{P}\left\{\tau_{x}>t\right\} \sim \mathbb{E}\left\{\tau_{x}\right\} \mathbb{P}\left\{X_{1}>t a\right\} \sim \mathbb{E}\left\{\tau_{x}\right\} \bar{\Pi}(t a) \text { as } t \rightarrow \infty, \\
\mathbb{P}\left\{v_{x}>n\right\} \sim \mathbb{E}\left\{v_{x}\right\} \mathbb{P}\left\{X_{1}>n a\right\} \text { as } n \rightarrow \infty
\end{gathered}
$$

Remark 3.1. Note that Theorem B.2 in Appendix B implies that in the case of a Lévy process, in order to verify the conditions of Theorem 3.1, instead of considering the random variable $X_{1}$, 
one may consider any random variable $\xi_{1}$ such that $\mathbb{P}\left\{\xi_{1}>t\right\}=\bar{F}(t) \sim \bar{\Pi}(t)$ as $t \rightarrow \infty$. Indeed, it is easy to prove that if conditions (3.1) and (3.2) hold for a random variable $\xi_{1}$, they also hold for a random variable $\eta_{1}$ such that $\mathbb{P}\left\{\xi_{1}>t\right\} \sim \mathbb{P}\left\{\eta_{1}>t\right\}$ as $t \rightarrow \infty$. This is useful as, when dealing with a Lévy process, the distribution of an individual increment is not always known explicitly.

Remark 3.2. Note that the conditions of Theorem 3.2 imply that $\bar{F}(y-\sqrt{y}) \sim \bar{F}(y)$. It, in turn, implies that $-\ln \bar{F}(y)=o(\sqrt{y})$. Thus, we again have the Weibull distribution with parameter $\frac{1}{2}$ as a boundary.

Remark 3.3. It follows from the results of [12] that regularly varying distributions and Weibulltype distributions with parameter smaller than $\frac{1}{2}$ satisfy the conditions of Theorem 3.1 .

Proof of Theorem 3.2. We first check the conditions of Theorem 2.2. It follows from Theorem 3.1 that $\mathbb{P}\left\{X_{n} \geq 0\right\} \sim \mathbb{P}\left\{X_{n+1} \geq 0\right\}$ and $\mathbb{P}\left\{X_{n} \geq y\right\} \sim \mathbb{P}\left\{X_{n} \geq 0\right\}$. It is then easy to check that $\mathbb{P}\left\{X_{n} \geq 0\right\} / n$ is a subexponential sequence.

It follows from Theorem 3.1 and Theorem 2.2 that

$$
\mathbb{P}\left\{X_{t} \geq 0\right\} \sim \mathbb{P}\left\{X_{[t]} \geq 0\right\}=\mathbb{P}\left\{X_{[t]}+[t] a \geq[t] a\right\} \sim[t] \mathbb{P}\left\{X_{1} \geq[t] a\right\}
$$

Hence,

$$
\mathbb{P}\left\{\tau_{x}>t\right\} \sim \mathbb{E}\left\{\tau_{x}\right\} \frac{\mathbb{P}\left\{X_{t} \geq 0\right\}}{t} \sim \mathbb{E}\left\{\tau_{x}\right\} \mathbb{P}\left\{X_{1} \geq[t] a\right\} \sim \mathbb{E}\left\{\tau_{x}\right\} \mathbb{P}\left\{X_{1}>t a\right\}
$$

This completes the proof.

We also present a direct corollary of Theorem 3.2.

Corollary 3.1. Consider the $\mathrm{M} / \mathrm{G} / 1$ system defined in the introduction. Let $\mathbb{E}\left\{B_{1}^{\kappa}\right\}<\infty$ and $\mathbb{E}\left\{A_{1}^{\kappa}\right\}<\infty$ for some $\kappa \in(1,2]$. Assume that the distribution of $B_{1}$ satisfies conditions (3.1) and (3.2). Then

$$
\mathbb{P}\{b p(x)>t\} \sim \frac{x}{\mathbb{E} A_{1}} \frac{1}{1-\rho} \mathbb{P}\left\{B_{1}>(1-\rho) t\right\}
$$

as $t \rightarrow \infty$ for any fixed $x>0$.

Proof. In the case of an $\mathrm{M} / \mathrm{G} / 1$ system, $X_{1}=\sum_{i=1}^{N(1)} X_{i}-1$ (see (1.2)). Hence, $\mathbb{E}\left\{X_{1}\right\}=$ $\rho-1$ and $\mathbb{P}\left(X_{1}>t\right) \sim \mathbb{P}\left(B_{1}>t\right) / \mathbb{E} A_{1}$ as $t \rightarrow \infty$. Theorem 3.2 yields

$$
\mathbb{P}\{b p(x)>t\} \sim \mathbb{E}\{b p(x)\} \mathbb{P}\left\{B_{1}>(1-\rho) t\right\}
$$

as $t \rightarrow \infty$. It remains to note that $\mathbb{E} b p(x)=x /(1-\rho)$ (this can be obtained from, e.g. [10, Equation (4.94), p. 261]). This completes the proof.

\subsection{Heavy-tailed distributions II}

Define $g(x)=-\ln \bar{F}(x)$. In this subsection we consider the case

$$
\limsup \frac{g(x)}{\sqrt{x}}>0 .
$$

For this, we use [28, Theorem 5a]. 
Theorem 3.3. ([28, Theorem 5a].) Let $\mathbb{P}\left\{\xi_{1}>y\right\} \sim \mathrm{e}^{-g(y)}$ as $y \rightarrow \infty$ with a doubly differentiable function $g$ such that $g^{\prime \prime}(y)$ does not decrease for $y \geq y_{o}$ and $y g^{\prime \prime}(y) \sim(\beta-1) g^{\prime}(y)$ as $y \rightarrow \infty$ for some $\beta \in(0,1)$. Let

$$
k=\max \left\{l \in\{1,2, \ldots\}: \limsup _{z \rightarrow \infty} \frac{g(z)}{z^{l /(l+1)}}>0\right\} .
$$

Let

$$
\begin{aligned}
& \mathbb{E}\{\xi\}=0, \quad \mathbb{E}\left\{\xi^{2}\right\}=1, \quad \mathbb{E}\left\{|\xi|^{k+3}\right\}<\infty, \\
& R(y)=g(y)+\frac{(t-y)^{2}}{2 n}-\sum_{i=1}^{k} \lambda_{i-1} \frac{(t-y)^{i+2}}{n^{i+1}} .
\end{aligned}
$$

Let $y_{*}$ be the maximal solution of $R^{\prime}(y)=0$. Then $y^{*} \leq t-\sqrt{n}$ and

$$
\mathbb{P}\left\{S_{n}>t\right\} \sim n \sqrt{\frac{1}{n R^{\prime \prime}\left(y_{*}\right)}} \exp \left\{-R\left(y_{*}\right)\right\} \quad \text { as } n \rightarrow \infty
$$

uniformly in $t>1.6 \eta(n)$, where $\eta(z)$ is such that $\eta^{2}(z) / g(\eta(z)) \sim z$ as $z \rightarrow \infty$. Here, the $\lambda_{i}$ are the coefficients of Cramér's series (see [26] for the definition).

Remark 3.4. Note that the conditions of Theorem 3.3 imply that $g^{\prime \prime}(y)$ is a regularly varying function with parameter $\beta-2$. This fact follows from the monotonicity of $g^{\prime \prime}$ and Karamata's theorem. Then $g^{\prime}(y)$ is regularly varying with parameter $\beta-1$ and $g(y)$ is regularly varying with parameter $\beta$. Also, under these conditions, $\eta(z)$ may be equivalently defined as a function such that $\left|g^{\prime \prime}(\eta(z))\right| \sim \beta(1-\beta) / z$ as $z \rightarrow \infty$. Therefore, $\eta(z)$ is a monotone regularly varying function with parameter $1 /(2-\beta)$.

Remark 3.5. In the statement of [28, Theorem 5a] it is not stated that $y^{*} \leq t-\sqrt{n}$, but one can find this assertion in the proof of [28, Lemma 3a].

We find it difficult to apply Theorem 3.3 directly, since it gives the asymptotics in terms of the maximal solution to an equation. Therefore, we use the approach developed in [17] to simplify this equation.

Lemma 3.1. Suppose that the conditions of Theorem 3.3 hold. Let $t_{n} \rightarrow \infty$ be a sequence such that $t_{n} \geq 1.6 \eta(n)$. Let $y_{n}$ be any sequence such that $y_{n} \sim t_{n}$ and

$$
R^{\prime}\left(y_{n}\right)=o\left(\frac{1}{\sqrt{n}}\right)
$$

Then

$$
\mathbb{P}\left\{S_{n}>t_{n}\right\} \sim n \sqrt{\frac{1}{n R^{\prime \prime}\left(y_{n}\right)}} \exp \left\{-R\left(y_{n}\right)\right\} \quad \text { as } n \rightarrow \infty .
$$

Also, for any sequence $\varepsilon_{n}=o(\sqrt{n})$,

$$
\mathbb{P}\left\{S_{n}>t_{n}\right\} \sim \mathbb{P}\left\{S_{n}>t_{n}+\varepsilon_{n}\right\}
$$


Proof. First, we note that, since $g^{\prime \prime}$ is monotone and regularly varying, it is true that

$$
R^{\prime}(y+z)-R^{\prime}(y)=z R^{\prime \prime}(y)(1+o(1)), \quad y \rightarrow \infty, z=o(y) .
$$

Also, $R^{\prime \prime}\left(y_{n}\right)=\left(g^{\prime \prime}\left(y_{n}\right)+1 / n\right)(1+o(1))$ and

$$
\begin{aligned}
\left|g^{\prime \prime}\left(y_{n}\right)\right| & \leq\left|g^{\prime \prime}\left(1+o(1) t_{n}\right)\right| \\
& \leq(1+o(1))\left|g^{\prime \prime}(1.6 \eta(n))\right| \\
& \leq(1+o(1))\left|g^{\prime \prime}(\eta(n))\right| \\
& =\frac{(1+o(1)) \beta(1-\beta)}{n} \\
& \leq \frac{1}{4 n} .
\end{aligned}
$$

Then

$$
\begin{aligned}
& R^{\prime}\left(y_{n}+\varepsilon \sqrt{n}\right)=R^{\prime}\left(y_{n}\right)+\varepsilon \sqrt{n} R^{\prime \prime}\left(y_{n}\right) \geq o\left(\frac{1}{\sqrt{n}}\right)+\frac{3 / 4 \varepsilon}{\sqrt{n}}>0, \\
& R^{\prime}\left(y_{n}-\varepsilon \sqrt{n}\right)=R^{\prime}\left(y_{n}\right)-\varepsilon \sqrt{n} R^{\prime \prime}\left(y_{n}\right) \leq o\left(\frac{1}{\sqrt{n}}\right)-\frac{1 / 4 \varepsilon}{\sqrt{n}}<0,
\end{aligned}
$$

for any $\varepsilon>0$. Since $R^{\prime}$ is continuous, there exists a sequence $\beta_{n} \in\left(y_{n}-o(\sqrt{n}), y_{n}+o(\sqrt{n})\right)$, such that $R^{\prime}\left(\beta_{n}\right)=0$ and $\beta_{n} \sim t_{n}$. Furthermore, if there exists another solution $\beta_{n}^{\prime}>\beta_{n}$ then, with necessity, $\beta_{n}^{\prime} \sim t_{n} \sim \beta_{n}$. But, this is not possible since $R^{\prime \prime}(y)$ is positive on the interval $\left(\beta_{n}, t_{n}\right)$.

To prove the first statement of the lemma, note that

$$
\begin{aligned}
R\left(y_{n}\right)-R\left(\beta_{n}\right) & =R^{\prime}\left(\beta_{n}\right)\left(\beta_{n}-y_{n}\right)+(1+o(1)) R^{\prime \prime}\left(\beta_{n}\right) \frac{\left(\beta_{n}-y_{n}\right)^{2}}{2} \\
& =(1+o(1)) R^{\prime \prime}\left(\beta_{n}\right) \frac{\left(\beta_{n}-y_{n}\right)^{2}}{2} \\
& \sim O\left(\frac{1}{n}\right) o(\sqrt{n})^{2} \\
& =o(1) .
\end{aligned}
$$

To prove the second statement of the lemma, we note that if $\beta_{n}$ is a solution sequence of the equation $R_{1}\left(\beta_{n}\right)=0$ for the first sequence $t_{n}$, then the corresponding equation for the sequence $t_{n}+\varepsilon_{n}$ is $R_{2}\left(\beta_{n}\right)=o(1 / \sqrt{n})$. Then we just apply the first statement of the lemma. This completes the proof.

We shall now concentrate on the case $t_{n}=n a$, the case needed for our purposes.

Corollary 3.2. Suppose that the conditions of Theorem 3.3 hold. Let $t_{n}=n a$, where $a>0$. Let $y_{n}$ be any sequence such that $y_{n} \sim t_{n}$, and assume that condition (3.4) holds. Then

$$
\mathbb{P}\left\{S_{n}>n a\right\} \sim n \exp \left\{-R\left(y_{n}\right)\right\} \text { as } n \rightarrow \infty .
$$

Also,

$$
\mathbb{P}\left\{S_{n}>n a\right\} \sim \mathbb{P}\left\{S_{n}>n a+\varepsilon_{n}\right\}
$$

for any sequence $\varepsilon_{n}=o(\sqrt{n})$. 
Proof. This is just a reformulation of Lemma 3.1. We only note that in this case $R^{\prime \prime}\left(y_{n}\right) \sim 1 / n$.

Lemma 3.2. Under the conditions of Theorem 3.3, let $t_{n}=n a$. Define a sequence

$$
y_{n}^{(0)}=n a, \quad y_{n}^{(j)}=y_{n}^{(j-1)}-n R^{\prime}\left(y_{n}^{(j-1)}\right) .
$$

Then

$$
\mathbb{P}\left\{S_{n}>n a\right\}=n \exp \left\{-R\left(y_{n}^{(j)}\right)\right\}
$$

as $n \rightarrow \infty$ for any $j \geq 1 /(2 k)$.

Proof. We have

$$
\left|y_{n}^{(1)}-y_{n}^{(0)}\right|=n\left|R^{\prime}(n a)\right|=n g^{\prime}(n a) .
$$

This implies that $y_{n}^{(2)} \sim n a$. Assume that we have proved that $y_{n}^{(i)} \sim n a$ for all $i<j$. Then, using regular variation of $g^{\prime \prime}$, we obtain

$$
\begin{aligned}
\left|y_{n}^{(j)}-y_{n}^{(j-1)}\right| & =n\left|R^{\prime}\left(y_{n}^{j-1}\right)-R^{\prime}\left(y_{n}^{j-2}\right)\right| \\
& =(1+o(1)) n\left|g^{\prime}\left(y_{n}^{j-1}\right)-g^{\prime}\left(y_{n}^{j-2}\right)\right| \\
& =(1+o(1)) n\left|g^{\prime \prime}(n a)\right|\left|y_{n}^{j-1}-y_{n}^{j-2}\right| \\
& =o(n) .
\end{aligned}
$$

Therefore, we can argue by induction that

$$
\left|y_{n}^{(j)}-y_{n}^{(j-1)}\right|=O(1)\left(n\left|g^{\prime \prime}(n a)\right|\right)^{j-1} n g^{\prime}(n a)=O(1) n\left(g^{\prime}(n)\right)^{j}=O(1) n\left(\frac{g(n)}{n}\right)^{j}
$$

for $j \geq 1$. By making use of condition (3.3), we obtain

$$
\left|y_{n}^{(j+1)}-y_{n}^{(j)}\right|=o(1) n\left(\frac{n^{1-1 /(k+2)}}{n}\right)^{j+1}=o(1) n^{1-(j+1) /(k+2)}=o(\sqrt{n}),
$$

provided $j \geq k / 2$. Then

$$
R^{\prime}\left(y_{n}^{(j)}\right)=\frac{y_{n}^{(j)}-y_{n}^{(j+1)}}{n}=\frac{o(\sqrt{n})}{n}=o\left(\frac{1}{\sqrt{n}}\right) .
$$

This completes the proof.

Lemma 3.3. Assume that the conditions of Lemma 3.2 hold. Then the sequence $a_{n}=$ $\mathbb{P}\left\{S_{n} \geq 0\right\} / n$ is subexponential.

Proof. It follows from Lemma 3.2 that $a_{n} \sim \mathrm{e}^{-R\left(y_{n}^{(j)}\right)}$ for sufficiently large $j$. We shall prove that

$$
\lim _{n \rightarrow \infty} \frac{n R^{\prime}\left(y_{n}^{(j)}\right)}{R\left(y_{n}^{(j)}\right)}=\beta
$$

for any $j$. This will imply that the sequence $a_{n}$ is subexponential due to the sufficient conditions given in [19]. We prove (3.7) by induction. If $j=0$ then $R\left(y_{n}^{(j)}\right)=g\left(y_{n}^{(j)}\right)$ and (3.7) holds since $g$ is a regularly varying function with parameter $\beta$. Assume that (3.7) holds for some $j$. 
Note that (3.6) implies that $R^{\prime}\left(y_{n}^{(j)}\right)=\left(y_{n}^{(j)}-y_{n}^{(j+1)}\right) / n$ is regularly varying, and, hence, taking (3.7) into account, $R\left(y_{n}^{(j)}\right)$ is also regularly varying. Recall that $y_{n}^{(j)} \sim n a$ for each $j$ and that $y_{n}^{(j)}-y_{n}^{(j+1)}=o(n)\left(\right.$ see (3.5)). Then $R\left(y_{n}^{(j+1)}\right)=R\left(y_{n}^{(j)}+\left(y_{n}^{(j+1)}-y_{n}^{(j)}\right)\right) \sim R\left(y_{n}^{(j)}\right)$ and also $R^{\prime}\left(y_{n}^{(j+1)}\right) \sim R^{\prime}\left(y_{n}^{(j)}\right)$. Hence, (3.7) holds for $j+1$ as well. This completes the proof.

We now give the result for the asymptotic behaviour of the tails of $\tau_{x}$ and $v_{x}$.

Theorem 3.4. Assume that either a Lévy process $\left\{X_{t}\right\}$ or a random walk $X_{n}$ is such that $\mathbb{E}\left\{X_{1}\right\}=-a<0$. Let the conditions of Theorem 3.3 hold for the distribution of the random variable $X_{1}+a$. Let $R^{\prime}(y(t))=o(1 / \sqrt{t})$. Then

$$
\begin{aligned}
\mathbb{P}\left\{\tau_{x}>t\right\} & \sim \mathbb{E}\left\{\tau_{x}\right\} \exp \{-R(y(t))\} \quad \text { as } t \rightarrow \infty, \\
\mathbb{P}\left\{v_{x}>n\right\} & \sim \mathbb{E}\left\{v_{x}\right\} \exp \{-R(y(n))\} \quad \text { as } n \rightarrow \infty
\end{aligned}
$$

Remark 3.6. Note that, as in the previous case, Theorem B.2 in Appendix B implies that in the case of a Lévy process, in order to verify the conditions of Theorem 3.3, instead of the random variable $X_{1}$, one may consider a random variable $\xi_{1}$ such that $\mathbb{P}\left\{\xi_{1}>t\right\}=\bar{F}(t) \sim \bar{\Pi}(t)$ as $t \rightarrow \infty$.

Proof of Theorem 3.4. We use Theorem 2.2 again. First, it follows from Corollary 3.2 that $\mathbb{P}\left\{X_{n}>y\right\} \sim \mathbb{P}\left\{X_{n}>0\right\} \sim \mathbb{P}\left\{X_{n+1}>0\right\}$. Second, according to Lemma 3.3, the sequence $\alpha_{n}=\mathbb{P}\left\{X_{n}>0\right\} / n$ is subexponential. Then we can just apply Theorem 2.2. This completes the proof.

Corollary 3.3. Let $\mathbb{P}\left\{B_{1}>y\right\} \sim \mathrm{e}^{-g(y)}$. Assume that $g$ and $B_{1}$ satisfy the conditions of Theorem 3.4 with $a=1-\rho$. Then the asymptotics are given by

$$
\mathbb{P}\{b p(x)>t\} \sim \frac{x}{\mathbb{E}\{A\}(1-\rho)} \exp \{-R(y(t))\} \quad \text { as } t \rightarrow \infty .
$$

Proof. The proof is a repetition of that of Corollary 3.1 and is thus omitted.

We can apply Lemma 3.2 to obtain tail asymptotics for the distributions of $\tau_{x}$ and $v_{x}$ explicitly. In particular, the following corollary holds.

Corollary 3.4. Under the conditions of Theorem 3.4, let $g(y)=o\left(y^{3 / 4}\right)$. Then

$$
\mathbb{P}\left\{\tau_{x}>t\right\} \sim \mathbb{E}\left\{\tau_{x}\right\} \exp \left\{-R\left(t a-\operatorname{tg}^{\prime}(t a)\right)\right\} \text { as } t \rightarrow \infty .
$$

If $\mathbb{P}\left\{B_{1}>t\right\} \sim \mathrm{e}^{-t^{\beta}}, \frac{1}{2}<\beta<1$, then, for $k=[1 /(1-\beta)]$ and some positive constants $D_{1}, \ldots, D_{k}>0$,

$$
\mathbb{P}\left\{\tau_{x}>t\right\} \sim \mathbb{E}\left\{\tau_{x}\right\} \exp \left\{-(a t)^{\beta}+D_{1} t^{2 \beta-1}+\cdots+D_{k} t^{k \beta-k+1}\right\} .
$$

Similar corollaries can be formulated for $v_{x}$ and $b p(x)$.

\subsection{Cramér's case}

Let $m(s)=\mathbb{E}\left\{\mathrm{e}^{s X_{1}}\right\}$ be the moment generating function of $X_{1}$.

Theorem 3.5. Assume that either a Lévy process $\left\{X_{t}\right\}$ or a random walk $X_{n}$ is such that $\mathbb{E}\left\{X_{1}\right\}=-a<0$. Let solution $\alpha$ to the equation $m^{\prime}(s)=0$ exist, and assume that $m^{\prime \prime}(\alpha)<\infty$. 
Put $\gamma=\ln m(\alpha)$. Assume also that the distribution of $X_{1}$ is nonlattice. Then

$$
\begin{gathered}
\mathbb{P}\left\{\tau_{x}>t\right\} \sim V(x) \frac{1}{\sqrt{2 \pi} t^{3 / 2} \widehat{\sigma}(\alpha) \alpha} \mathrm{e}^{-\gamma t} \text { as } t \rightarrow \infty, \\
\mathbb{P}\left\{v_{x}>n\right\} \sim \frac{1}{\sqrt{2 \pi} n^{3 / 2} \widehat{\sigma}(\alpha) \alpha} \mathrm{e}^{-\gamma n} \text { as } n \rightarrow \infty .
\end{gathered}
$$

Proof. It follows from the Petrov theorem (see [25, Theorem 2]) that $a_{n}=\mathbb{P}\left\{X_{n} \geq 0\right\} / n \sim$ $\mathrm{e}^{-\gamma n} /\left(\sqrt{2 \pi} n^{3 / 2} \widehat{\sigma}(\alpha) \alpha\right)$. Then $a_{n} \in s_{s}(\gamma)$. Indeed, $a_{n-1} / a_{n} \rightarrow \mathrm{e}^{\gamma}$ and

$$
\sum_{k=1}^{n-1} \frac{a_{k} a_{n-k}}{a_{n}} \leq C \sum_{k=1}^{n-1}\left(\frac{n}{k(n-k)}\right)^{3 / 2} \leq 4 C \sum_{k=1}^{n-1} \frac{1}{k^{3 / 2}}<4 C \sum_{k=1}^{\infty} \frac{1}{k^{3 / 2}}
$$

for some constant $C$. It follows from the dominated convergence theorem that $a_{n}^{* 2} / a_{n} \rightarrow$ $2 \sum_{n=1}^{\infty} \mathrm{e}^{\gamma n} a_{n}$. Also, Petrov's theorem implies that

$$
\frac{\mathbb{P}\left\{X_{n} \geq y\right\}}{\mathbb{P}\left\{X_{n} \geq 0\right\}} \sim \mathrm{e}^{-\alpha y} \quad \text { as } n \rightarrow \infty .
$$

Therefore, we can apply Theorem 2.2 to obtain the statement of the theorem.

Corollary 3.5. Let $\alpha>0$ be a solution to the equation $\lambda m_{B}^{\prime}(\alpha)=1$ such that $\widehat{\sigma}^{2}=$ $\lambda m_{B}^{\prime \prime}(\alpha)<\infty$. Put $\gamma=\alpha-\lambda\left(m_{B}(\alpha)-1\right)$. Then

$$
\mathbb{P}\{b p(x)>t\} \sim \frac{1}{\sqrt{2 \pi \hat{\sigma}^{2}} \gamma t^{3 / 2}} x \mathrm{e}^{\alpha x} \mathrm{e}^{-\gamma t} \text { as } t \rightarrow \infty .
$$

Proof. It is clear that $\alpha$ and $\gamma$ are exactly the same as in Theorem 3.5. Therefore, it suffices to find $V(x)$. Since $\mathrm{e}^{-\gamma}=\mathbb{E}\left\{\mathrm{e}^{\alpha X_{1}}\right\}$, the process $\exp \left\{\alpha X_{t}+\gamma t\right\}$ is a martingale with mean 1 . Then, since $X_{\tau_{x}}=-x$, we have

$$
1=\mathbb{E}\left\{\mathrm{e}^{\alpha X_{\tau_{x}}+\gamma \tau_{x}}\right\}=\mathrm{e}^{-\alpha x} \mathbb{E}\left\{\mathrm{e}^{\gamma \tau_{x}}\right\} .
$$

Hence, $C(x)=\gamma^{-1}\left(\mathbb{E}\left\{\mathrm{e}^{\gamma \tau_{x}}\right\}-1\right)=\gamma^{-1}\left(\mathrm{e}^{\alpha x}-1\right)$, and it follows from (A.7) below that

$$
V(x)=C(x)+\alpha \mathrm{e}^{\alpha x} \int_{0}^{x} \mathrm{e}^{-\alpha y} C(y) \mathrm{d} y=\frac{\alpha}{\gamma} x \mathrm{e}^{\alpha x} .
$$

This completes the proof.

\subsection{Intermediate case}

We now proceed to the intermediate case, i.e. when the equation $m^{\prime}(s)=0$ does not have a positive solution but $m(s)<\infty$ for some $s>0$. In this case we shall assume that $\mathbb{P}\left\{\xi_{1}>t\right\}=$ $\mathrm{e}^{-\alpha t} \bar{G}(t)$ for all positive $t$, where $\alpha>0$ and $\bar{G}(t)$ is a tail of some heavy-tailed distribution. Introduce the random walk $\left\{\tilde{S}_{n}\right\}$ (called the adjunct random walk in [5]) whose increments have the distribution

$$
\tilde{F}(\mathrm{~d} y)=\frac{1}{m(\alpha)} \mathrm{e}^{\alpha y} F(\mathrm{~d} y),
$$

and define $\delta=-\mathbb{E}\left\{\tilde{S}_{1}\right\}$. The following result on large deviations may be found in [12]. It is a generalization of the result of [5] where asymptotics for the large deviation probabilities are obtained under the assumption that $\bar{G}(t)$ is a regularly varying function. 
Theorem 3.6. Assume that $\mathbb{P}\left\{\xi_{1}>t\right\}=\mathrm{e}^{-\alpha t} \bar{G}(t)$ for all positive $t$, where $\alpha>0$ and $\bar{G}(t)$ satisfies the conditions of Theorem 3.1. Let $\mathbb{E}\left\{\xi_{1}\right\}<0$, and assume that $m^{\prime}(s) \neq 0$ for $0<s \leq \alpha$. Assume also that $\delta<\infty$. Put $\mathrm{e}^{-\gamma}=m(\alpha)$. Then

$$
\mathbb{P}\left\{S_{n}>x\right\} \sim \frac{1}{m(\alpha)} \mathrm{e}^{-\gamma n} \mathrm{e}^{-\alpha x} n \bar{G}(x+n \delta)
$$

as $n \rightarrow \infty$ uniformly in $x$ such that $x \geq-n(\delta-\varepsilon)$.

Remark 3.7. It is easy to see that the conditions of Theorem 3.6 imply that $\delta>0$.

Using Theorem 3.6, we can obtain the following result for the tail asymptotics of $\tau_{x}$ and $v_{x}$.

Theorem 3.7. Assume that $\left\{X_{t}\right\}$ is a Lévy process or a random walk such that the distribution of $X_{1}$ satisfies the conditions of Theorem 3.6. Then

and

$$
\mathbb{P}\left\{\tau_{x}>t\right\} \sim V(x) \frac{1}{m(\alpha)} \mathrm{e}^{-\gamma t} \bar{G}(t \delta) \quad \text { as } t \rightarrow \infty
$$

$$
\mathbb{P}\left\{v_{x}>n\right\} \sim V_{r w}(x) \frac{1}{m(\alpha)} \mathrm{e}^{-\gamma n} \bar{G}(n \delta) \text { as } n \rightarrow \infty .
$$

Proof. Theorem 3.6 implies that

$$
\mathbb{P}\left\{X_{n} \geq 0\right\} \sim \frac{1}{m(\alpha)} \mathrm{e}^{-\gamma n} n \bar{G}(n \delta) \quad \text { and } \quad \mathbb{P}\left\{X_{n} \geq y\right\} \sim \frac{1}{m(\alpha)} \mathrm{e}^{-\gamma n} n \bar{G}(n \delta+y) \mathrm{e}^{-y \alpha}
$$

as $n \rightarrow \infty$. The conditions of Theorem 2.2 can now be checked straightforwardly.

Remark 3.8. To the best of our knowledge, the only result on large deviations of sums of random variables belonging to the intermediate case is contained in [5]. The result presented in this paper concerns distributions $F$ such that the function $\mathrm{e}^{\alpha x} \bar{F}(x)$ is regularly varying with parameter $-\beta, 2<\beta<\infty$. The analogue of Theorem 3.7 for such distributions may be obtained using Lemma 3 from [5] instead of our Theorem 3.6.

\section{Appendix A. Proofs of Theorems 2.1 and 2.2}

Proof of Result 2.1. We first prove that $\mathbb{E}\left\{\mathrm{e}^{\alpha X_{1}}\right\} \leq \mathrm{e}^{-\gamma}$. Fix an arbitrary $C>0$. Then

$$
\mathrm{e}^{\alpha y} \leq(1+\varepsilon) \frac{\left.\mathbb{P}\left\{X_{t}>-y\right)\right\}}{\mathbb{P}\left\{X_{t}>0\right\}}
$$

for large enough $t$, uniformly in $y \in(-C, C)$. Consider, for large enough $t$,

$$
\begin{aligned}
\int_{-C}^{C} \mathrm{e}^{\alpha y} \mathbb{P}\left\{X_{1} \in \mathrm{d} y\right\} & \leq(1+\varepsilon) \frac{\int_{-C}^{C} \mathbb{P}\left\{X_{t}>-y\right\} \mathbb{P}\left\{X_{1} \in \mathrm{d} y\right\}}{\mathbb{P}\left\{X_{t}>0\right\}} \\
& =(1+\varepsilon) \frac{\int_{-C}^{C} \mathbb{P}\left\{X_{t}>-y\right\} \mathbb{P}\left\{X_{t+1}-X_{t} \in \mathrm{d} y\right\}}{\mathbb{P}\left\{X_{t}>0\right\}} \\
& =(1+\varepsilon) \frac{\mathbb{P}\left\{X_{t+1}-X_{t} \in(-C, C), X_{t+1}>0\right\}}{\mathbb{P}\left\{X_{t}>0\right\}} \\
& \leq \frac{\mathbb{P}\left\{X_{t+1}>0\right\}}{\mathbb{P}\left\{X_{t}>0\right\}} \\
& \leq(1+\varepsilon)^{2} \mathrm{e}^{-\gamma} .
\end{aligned}
$$


Since $\varepsilon$ is an arbitrary positive number, we have $\int_{-C}^{C} \mathrm{e}^{\alpha y} \mathbb{P}\left\{X_{1} \in \mathrm{d} y\right\} \leq \mathrm{e}^{-\gamma}$ and, hence, $\mathbb{E}\left\{\mathrm{e}^{\alpha X_{1}}\right\} \leq \mathrm{e}^{-\gamma}$.

The inequality $\mathbb{E}\left\{\mathrm{e}^{\alpha X_{1}}\right\} \geq \mathrm{e}^{-\gamma}$ can be proved in a similar way. Take an arbitrary $C>0$ again and consider

$$
\int_{-C}^{C} \frac{\mathbb{P}\left\{X_{t}>-y\right\} \mathbb{P}\left\{X_{t+1}-X_{t} \in \mathrm{d} y\right\}}{\mathbb{P}\left\{X_{t}>0\right\}} \leq(1+\varepsilon) \int_{-C}^{C} \mathrm{e}^{\alpha y} \mathbb{P}\left\{X_{1} \in \mathrm{d} y\right\} \leq(1+\varepsilon) \mathbb{E}\left\{\mathrm{e}^{\alpha X_{1}}\right\}
$$

for sufficiently large $t$. Since $C$ is arbitrary, we have

$$
\frac{\mathbb{P}\left\{X_{t+1}>0\right\}}{\mathbb{P}\left\{X_{t}>0\right\}}=\int_{-\infty}^{\infty} \frac{\mathbb{P}\left\{X_{t}>-y\right\} \mathbb{P}\left\{X_{t+1}-X_{t} \in \mathrm{d} y\right\}}{\mathbb{P}\left\{X_{t}>0\right\}} \leq(1+\varepsilon) \mathbb{E}\left\{\mathrm{e}^{\alpha X_{1}}\right\} .
$$

We also have

$$
\mathrm{e}^{-\gamma} \leq(1+\varepsilon) \frac{\mathbb{P}\left\{X_{t+1}>0\right\}}{\mathbb{P}\left\{X_{t}>0\right\}} \leq(1+\varepsilon)^{2} \mathbb{E}\left\{\mathrm{e}^{\alpha X_{1}}\right\}
$$

for sufficiently large $t$, which concludes the proof since the left-hand side does not depend on $\varepsilon$.

\section{A.1. Proof of Theorem 2.1}

Recall that $N_{t}=\inf _{s \leq t} X_{s}$. It is clear that $\mathbb{P}\left\{\tau_{x}>t\right\}=\mathbb{P}\left\{\left|N_{t}\right| \leq x\right\}$. Our starting point is the formula that follows from the Wiener-Hopf identity for Lévy processes; see [29, Equation (47.9)] (with obvious changes: we should substitute the infimum process instead of the supremum process). Thus, for $q>0$ and $u \geq 0$,

$$
q \int_{0}^{\infty} \mathrm{e}^{-q t} \mathbb{E}\left\{\mathrm{e}^{u N_{t}}\right\} \mathrm{d} t=\exp \left\{\int_{0}^{\infty} t^{-1} \mathrm{e}^{-q t} \mathrm{~d} t \int_{(-\infty, 0)}\left(\mathrm{e}^{u y}-1\right) \mathbb{P}\left\{X_{t} \in \mathrm{d} y\right\}\right\} .
$$

For $q>0$, use the Frullani integral,

$$
-\ln q=\int_{0}^{\infty} \frac{\mathrm{e}^{-q t}-\mathrm{e}^{-t}}{t} \mathrm{~d} t
$$

and rewrite (A.1) in the form

$$
\begin{aligned}
\int_{0}^{\infty} \mathrm{e}^{-q t} \mathbb{E}\left\{\mathrm{e}^{u N_{t}}\right\} \mathrm{d} t=\exp & \left\{\int_{0}^{\infty} \frac{\mathrm{e}^{-q t}-\mathrm{e}^{-t}}{t} \mathrm{~d} t\right. \\
& \left.+\int_{0}^{\infty} t^{-1} \mathrm{e}^{-q t} \mathrm{~d} t \int_{(-\infty, 0)}\left(\mathrm{e}^{u y}-1\right) \mathbb{P}\left\{X_{t} \in \mathrm{d} y\right\}\right\} .
\end{aligned}
$$

First we want to show that the right-hand side of (A.2) converges as $q \rightarrow 0$. To this end, let us represent the exponent on the right-hand side of (A.2) as

$$
\begin{aligned}
& \int_{0}^{1} \frac{\mathrm{e}^{-q t}-1}{t} \mathrm{~d} t+\int_{0}^{1} \frac{1-\mathrm{e}^{-t}}{t} \mathrm{~d} t-\int_{1}^{\infty} \frac{\mathrm{e}^{-t}}{t} \mathrm{~d} t \\
& +\int_{1}^{\infty} t^{-1} \mathrm{e}^{-q t}\left\{1+\int_{(-\infty, 0)}\left(\mathrm{e}^{u y}-1\right) \mathbb{P}\left\{X_{t} \in \mathrm{d} y\right\}\right\} \mathrm{d} t \\
& +\int_{0}^{1} t^{-1} \mathrm{e}^{-q t} \mathrm{~d} t \int_{(-\infty, 0)}\left(\mathrm{e}^{u y}-1\right) \mathbb{P}\left(X_{t} \in \mathrm{d} y\right) .
\end{aligned}
$$


It is clear that the first integral converges to 0 as $q \rightarrow 0$. The second and third integrals are well defined and constant. The fourth and fifth integrals are monotone in $q$, and, therefore, it is sufficient to prove that they are finite for $q=0$ and then to apply the monotone convergence theorem. For $q=0$, the fourth integral is equal to

$$
\begin{aligned}
\int_{1}^{\infty} t^{-1}\left\{1+\int_{(-\infty, 0)}\left(\mathrm{e}^{u y}-1\right) \mathbb{P}\left\{X_{t} \in \mathrm{d} y\right\}\right\} \mathrm{d} t \\
\quad=\int_{1}^{\infty} t^{-1}\left\{\mathbb{P}\left\{X_{t} \geq 0\right\}+\int_{(-\infty, 0)} \mathrm{e}^{u y} \mathbb{P}\left\{X_{t} \in \mathrm{d} y\right\}\right\} \mathrm{d} t .
\end{aligned}
$$

To deal with the second term in (A.4), we need the following result.

Lemma A.1. Let the function $\mathbb{P}\left\{X_{t} \geq 0\right\} / t$ be such that condition (2.1) holds. Assume also that condition (2.4) of Theorem 2.1 holds. Then there exists $u_{0}$ such that

$$
\int_{(-\infty, 0)} \mathrm{e}^{u y} \mathbb{P}\left\{X_{t} \in \mathrm{d} y\right\} \sim \frac{\alpha}{u-\alpha} \mathbb{P}\left\{X_{t} \geq 0\right\}
$$

for any $u>u_{0}$.

Remark. We use the convention that $a(x) \sim 0 \cdot b(x)$ means that $a(x)=o(b(x))$.

Proof of Lemma A.1. Using integration by parts, we obtain

$$
\int_{(-\infty, 0)} \mathrm{e}^{u y} \mathbb{P}\left\{X_{t} \in \mathrm{d} y\right\}=u \int_{0}^{\infty}\left(\mathbb{P}\left\{X_{t} \geq-y\right\}-\mathbb{P}\left\{X_{t} \geq 0\right\}\right) \mathrm{e}^{-u y} \mathrm{~d} y .
$$

We can a choose a function $h(t) \uparrow \infty$ such that (2.4) holds uniformly in $y \in[-h(t), 0]$. Then

$$
\begin{aligned}
u \int_{0}^{h(t)} & \left(\mathbb{P}\left\{X_{t} \geq-y\right\}-\mathbb{P}\left\{X_{t} \geq 0\right\}\right) \mathrm{e}^{-u y} \mathrm{~d} y \\
= & (1+o(1)) \mathbb{P}\left\{X_{t} \geq 0\right\} u \int_{0}^{h(t)}\left(\mathrm{e}^{\alpha y}-1\right) \mathrm{e}^{-u y} \mathrm{~d} y \\
= & \frac{\alpha+o(1)}{u-\alpha} \mathbb{P}\left\{X_{t} \geq 0\right\}
\end{aligned}
$$

for $u>\alpha$. Furthermore, since $\mathbb{P}\left\{X_{t} \geq 0\right\} / t$ satisfies condition (2.1),

$$
\mathbb{P}\left\{X_{t+n} \geq 0\right\} \leq \mathrm{e}^{n} \mathbb{P}\left\{X_{t} \geq 0\right\}, \quad n \geq 1,
$$

for all $n \geq 1$ and sufficiently large $t$. Since $\mathbb{P}\left\{X_{1}>0\right\}>0$, then $\mathbb{P}\left\{X_{1} \geq \delta\right\}>0$, where $\delta=1 / l>0$ with a positive integer $l$. Then

$$
\mathbb{P}\left\{X_{l n} \geq n\right\} \geq \mathbb{P}\left\{X_{1} \geq \delta, X_{2}-X_{1} \geq \delta, \ldots, X_{l n}-X_{l n-1} \geq \delta\right\}=\mathbb{P}\left\{X_{1} \geq \delta\right\}^{l n} .
$$

Let $u_{0}$ be such that $\mathrm{e}^{-u_{0}}=\mathrm{e}^{-2 l} \mathbb{P}\left\{X_{1} \geq \delta\right\}^{l}$. Then, for all $u>u_{0}$,

$$
\mathrm{e}^{-u n} \leq \mathrm{e}^{-u_{0} n}=\mathrm{e}^{-2 \ln } \mathbb{P}\left\{X_{1} \geq \delta\right\}^{l n} \leq \mathrm{e}^{-2 \ln } \mathbb{P}\left\{X_{l n} \geq n\right\}
$$


Therefore, for $u>u_{0}$,

$$
\begin{aligned}
\int_{-\infty}^{-h(t)}\left(\mathbb{P}\left\{X_{t} \geq-y\right\}-\mathbb{P}\left\{X_{t} \geq 0\right\}\right) \mathrm{e}^{-u y} \mathrm{~d} y & \leq \sum_{n=[h(t)]}^{\infty} \mathrm{e}^{-u n} \mathbb{P}\left\{X_{t} \geq-n\right\} \\
& \leq \sum_{n=[h(t)]}^{\infty} \mathrm{e}^{-2 \ln } \mathbb{P}\left\{X_{l n} \geq n\right\} \mathbb{P}\left\{X_{t} \geq-n\right\} \\
& =\sum_{n=[h(t)]}^{\infty} \mathrm{e}^{-2 \ln } \mathbb{P}\left\{X_{t+l n}-X_{t} \geq n, X_{t} \geq-n\right\} \\
& \leq \sum_{n=[h(t)]}^{\infty} \mathrm{e}^{-2 \ln } \mathbb{P}\left\{X_{t+l n} \geq 0\right\} .
\end{aligned}
$$

It follows from (A.5) that

$$
\sum_{n=[h(t)]}^{\infty} \mathrm{e}^{-2 \ln } \mathbb{P}\left\{X_{t+l n} \geq 0\right\} \leq \mathbb{P}\left\{X_{t} \geq 0\right\} \sum_{n=[h(t)]}^{\infty} \mathrm{e}^{-l n}=o\left(\mathbb{P}\left\{X_{t} \geq 0\right\}\right)
$$

as $t \rightarrow \infty$. This completes the proof of Lemma A.1.

We may now continue to analyse (A.4). It follows from Lemma A.1 that

$$
\int_{1}^{\infty} t^{-1}\left\{\mathbb{P}\left\{X_{t} \geq 0\right\}+\int_{(-\infty, 0)} \mathrm{e}^{u y} \mathbb{P}\left\{X_{t} \in \mathrm{d} y\right\}\right\} \mathrm{d} t \leq C \int_{1}^{\infty} t^{-1} \mathbb{P}\left\{X_{t} \geq 0\right\} \mathrm{d} t<\infty
$$

for some constant $C>0$. The finiteness of the latter integral follows from (1.1). We now proceed to the last term in (A.3). Making use of the inequality $1-\mathrm{e}^{-x} \leq x$ for all $x \geq 0$, we obtain

$$
\begin{aligned}
\int_{0}^{1} t^{-1} \mathrm{~d} t \int_{(-\infty, 0)}\left(1-\mathrm{e}^{u y}\right) \mathbb{P}\left\{X_{t} \in \mathrm{d} y\right\} \\
\quad \leq \int_{0}^{1} t^{-1} \mathrm{~d} t\left(\int_{(-1,0)}(-u y) \mathbb{P}\left\{X_{t} \in \mathrm{d} y\right\}+\mathbb{P}\left\{X_{t}<-1\right\}\right) \\
\quad=\int_{0}^{1} \mathbb{E}\left\{u X_{t} \mathbf{1}\left(X_{t} \in[-1,0]\right)\right\} t^{-1} \mathrm{~d} t+\int_{0}^{1} t^{-1} \mathrm{~d} t \mathbb{P}\left\{X_{t}<-1\right\} \\
\quad<\infty .
\end{aligned}
$$

The finiteness of the latter integral follows from the estimates in Lemma B.1 (see Appendix B). Therefore, the last term in (A.3) converges by the monotone convergence theorem.

Now, letting $q \rightarrow 0$ in (A.2), we have

$$
\int_{0}^{\infty} \mathbb{E}\left\{\mathrm{e}^{u N_{t}}\right\} \mathrm{d} t<\infty
$$

For a fixed $u$, let

$$
f_{u}(t)=\frac{\mathbb{E}\left\{\mathrm{e}^{u N_{t}}\right\}}{\int_{0}^{\infty} \mathbb{E}\left\{\mathrm{e}^{u N_{t}}\right\} \mathrm{d} t}
$$

be the density of a random variable $Z$. Then, using representations (A.3) and (A.4), we may 
rewrite (A.2) as

$$
\begin{aligned}
\mathbb{E}\left\{\mathrm{e}^{-q Z}\right\}= & \exp \left\{\int_{0}^{1} \frac{\mathrm{e}^{-q t}-1}{t} \mathrm{~d} t+\int_{1}^{\infty} \frac{\mathrm{e}^{-q t}-1}{t}\left(\mathbb{P}\left\{X_{t} \geq 0\right\}+\int_{(-\infty, 0)} \mathrm{e}^{u y} \mathbb{P}\left\{X_{t} \in \mathrm{d} y\right\}\right) \mathrm{d} t\right. \\
& \left.+\int_{0}^{1} \frac{\mathrm{e}^{-q t}-1}{t} \mathrm{~d} t \int_{(-\infty, 0)}\left(\mathrm{e}^{u y}-1\right) \mathbb{P}\left\{X_{t} \in \mathrm{d} y\right\}\right\} \\
\equiv & \exp \left\{\int_{0}^{1}\left(\mathrm{e}^{-q t}-1\right) \nu_{1}(\mathrm{~d} t)+\int_{1}^{\infty}\left(\mathrm{e}^{-q t}-1\right) \nu_{2}(\mathrm{~d} t)+\int_{0}^{1}\left(\mathrm{e}^{-q t}-1\right) \nu_{3}(\mathrm{~d} t)\right\} .
\end{aligned}
$$

It follows that $Z$ is an infinitely divisible variable on $[0, \infty)$ with the Lévy measure $v(\mathrm{~d} t)=$ $v_{1}(\mathrm{~d} t)+v_{2}(\mathrm{~d} t)+v_{3}(\mathrm{~d} t)$. Indeed, $\int_{0}^{1} t v_{1}(\mathrm{~d} t)<\infty$. Furthermore, as we have already shown, $v_{3}(0,1)<\infty$. Finally, as follows from Lemma A.1,

$$
f_{2}(t) \equiv \frac{\mathrm{d} \nu_{2}}{\mathrm{~d} t}=\frac{\mathbb{P}\left\{X_{t} \geq 0\right\}}{t}+\frac{1}{t} \int_{(-\infty, 0)} \mathrm{e}^{u y} \mathbb{P}\left\{X_{t} \in \mathrm{d} y\right\} \sim \frac{u}{u-\alpha} \frac{\mathbb{P}\left\{X_{t} \geq 0\right\}}{t} \quad \text { as } t \rightarrow \infty
$$

Therefore, by condition $(1.1), v_{2}(1, \infty)<\infty$. Now we are in the position to apply Theorem B.3 in Appendix B. Since the density of the Lévy measure $f(t) \sim \mathbb{P}\left\{X_{t} \geq 0\right\} / t$ belongs to the class $\delta d(\gamma)$, by Theorem B.3 we have

$$
f_{u}(t) \sim\left(\int_{0}^{\infty} \mathrm{e}^{\gamma y} f_{u}(y) \mathrm{d} y\right) \frac{u}{u-\alpha} \frac{\mathbb{P}\left\{X_{t} \geq 0\right\}}{t}
$$

for any fixed $u$. Equivalently,

$$
\frac{\mathbb{E}\left\{\mathrm{e}^{u N_{t}}\right\}}{\mathbb{P}\left\{X_{t} \geq 0\right\} / t} \rightarrow \frac{u}{u-\alpha} \int_{0}^{\infty} \mathrm{e}^{\gamma t} \mathbb{E}\left\{\mathrm{e}^{u N_{t}}\right\} \mathrm{d} t
$$

for all $u$. Then, changing the order of integration, we obtain

$$
\int_{0}^{\infty} \mathrm{e}^{\gamma t} \mathbb{E}\left\{\mathrm{e}^{u N_{t}}\right\} \mathrm{d} t=\int_{0}^{\infty} \mathrm{e}^{\gamma t}\left(\int_{0}^{\infty} \mathrm{e}^{-u x} \mathbb{P}\left\{\left|N_{t}\right| \in \mathrm{d} x\right\}\right) \mathrm{d} t=\int_{0}^{\infty} \mathrm{e}^{-u x} \mathrm{~d}_{x} C(x),
$$

where $C(x)=\int_{0}^{\infty} \mathrm{e}^{\gamma t} \mathbb{P}\left\{\left|N_{t}\right| \leq x\right\} \mathrm{d} t$. Therefore, (A.6) is equivalent to

$$
\frac{\mathbb{E}\left\{\mathrm{e}^{u N_{t}}\right\}}{\mathbb{P}\left\{X_{t} \geq 0\right\} / t} \rightarrow \frac{u}{u-\alpha} \int_{0}^{\infty} \mathrm{e}^{-u x} \mathrm{~d}_{x} C(x)
$$

as $t \rightarrow \infty$. Now note that $u /(u-\alpha)$ is the Laplace-Stieltjes transform of the measure $D$ which has unit mass at 0 and density $\alpha \mathrm{e}^{\alpha y}$ on the positive half-line. Therefore, for all $x$,

$$
\begin{aligned}
\frac{\mathbb{P}\left\{\left|N_{t}\right| \leq x\right\}}{\mathbb{P}\left\{X_{t} \geq 0\right\} / t} & \rightarrow D * C(x) \\
& =V(x) \\
& =C(x)+\alpha \mathrm{e}^{\alpha x} \int_{0}^{x} \mathrm{e}^{-\alpha y} C(y) \mathrm{d} y \\
& =\mathrm{e}^{\alpha x} \int_{0}^{\infty} \mathrm{e}^{\gamma t} \mathbb{E}\left\{\mathrm{e}^{\alpha N_{t}} ;\left|N_{t}\right| \leq x\right\} \mathrm{d} t,
\end{aligned}
$$


where the function $V(x)$ is continuous. This is equivalent to

$$
\frac{\mathbb{P}\left\{\tau_{x}>t\right\}}{\mathbb{P}\left\{X_{t} \geq 0\right\} / t} \rightarrow V(x)
$$

as $t \rightarrow \infty$. Finally, it is clear that if $\alpha=\gamma=0$ then

$$
V(x)=\int_{0}^{\infty} \mathbb{P}\left\{\left|N_{t}\right| \leq x\right\} \mathrm{d} t=\int_{0}^{\infty} \mathbb{P}\left\{\tau_{x}>t\right\} \mathrm{d} t=\mathbb{E}\left\{\tau_{x}\right\} .
$$

This completes the proof of Theorem 2.1.

Now we will show that for Theorem 2.1 to hold it is sufficient that its conditions hold for positive integers $t$. This will allow us to reduce the problem of verifying properties of $X_{t}, t \in \mathbb{R}$, to verifying the corresponding properties of the random walk $X_{n}, n \in \mathbb{N}$.

Lemma A.2. Assume that the sequence $\mathbb{P}\left\{X_{n} \geq 0\right\} / n, n \in \mathbb{N}$, belongs to the class $8 s(\gamma)$,

$$
\mathbb{P}\left(X_{n} \geq 0\right) \sim \mathrm{e}^{\alpha y} \mathbb{P}\left(X_{n} \geq y\right) \text { as } n \rightarrow \infty,
$$

for any fixed $y$, and $\mathrm{e}^{-\gamma}=\mathbb{E}\left\{\mathrm{e}^{\alpha X_{1}}\right\}$. Then the function $\mathbb{P}\left\{X_{t} \geq 0\right\} / t, t \in \mathbb{R}$, belongs to the class $s d(\gamma)$ and

$$
\mathbb{P}\left(X_{t} \geq 0\right) \sim \mathrm{e}^{\alpha y} \mathbb{P}\left(X_{t} \geq y\right)
$$

as $t \rightarrow \infty$ for any fixed $y$.

Proof. First, we prove that

$$
\mathbb{P}\left\{X_{n+\varepsilon} \geq 0\right\} \sim \mathrm{e}^{-\gamma \varepsilon} \mathbb{P}\left\{X_{n} \geq 0\right\}
$$

as $n \rightarrow \infty$ for any $0<\varepsilon<1$. It is not difficult to prove that there exists a function $h(n) \uparrow \infty$ such that condition (A.8) holds uniformly in $|z| \leq h(n)$. We start with the total probability formula

$$
\begin{aligned}
\mathbb{P}\left\{X_{n+\varepsilon} \geq 0\right\} \equiv & P_{1}+P_{2}+P_{3} \\
= & \mathbb{P}\left\{X_{n+\varepsilon} \geq 0,\left|X_{n+\varepsilon}-X_{n}\right| \leq h(n)\right\}+\mathbb{P}\left\{X_{n+\varepsilon} \geq 0, X_{n+\varepsilon}-X_{n}>h(n)\right\} \\
& +\mathbb{P}\left\{X_{n+\varepsilon} \geq 0, X_{n+\varepsilon}-X_{n}<-h(n)\right\} .
\end{aligned}
$$

Then, since $\mathbb{E}\left\{\mathrm{e}^{\alpha X_{1}}\right\}=\mathrm{e}^{-\gamma}$,

$$
\begin{aligned}
P_{1} & =\int_{-h(n)}^{h(n)} \mathbb{P}\left\{X_{\varepsilon} \in \mathrm{d} y\right\} \mathbb{P}\left\{X_{n} \geq-y\right\} \\
& \sim \mathbb{P}\left\{X_{n} \geq 0\right\} \int_{-\infty}^{\infty} \mathrm{e}^{\alpha y} \mathbb{P}\left\{X_{\varepsilon} \in \mathrm{d} y\right\} \\
& =\mathrm{e}^{-\gamma \varepsilon} \mathbb{P}\left\{X_{n} \geq 0\right\} .
\end{aligned}
$$

Before proceeding further, note that if we take $\varepsilon=1$ then it follows from (2.3), (A.11), and the latter equivalence that

$$
\mathbb{P}\left\{X_{n+1} \geq 0, X_{n+1}-X_{n}>h(n)\right\}=o\left(\mathbb{P}\left\{X_{n} \geq 0\right\}\right) .
$$

Furthermore,

$$
P_{2}=\mathbb{P}\left\{X_{n} \geq-h(n)\right\} \mathbb{P}\left\{X_{n+\varepsilon}-X_{n}>h(n)\right\}+\int_{-\infty}^{-h(n)} \mathbb{P}\left\{X_{n} \in \mathrm{d} y\right\} \mathbb{P}\left\{X_{n+\varepsilon}-X_{n} \geq-y\right\} .
$$


Now note that

$$
\mathbb{P}\left\{X_{1} \geq y\right\} \geq \mathbb{P}\left\{X_{\varepsilon} \geq y, X_{1}-X_{\varepsilon} \geq 0\right\}=\mathbb{P}\left\{X_{\varepsilon} \geq y\right\} \mathbb{P}\left\{X_{1-\varepsilon} \geq 0\right\},
$$

which implies that

$$
\begin{aligned}
P_{2} \leq & \frac{1}{\mathbb{P}\left\{X_{1-\varepsilon} \geq 0\right\}}\left(\mathbb{P}\left\{X_{n} \geq-h(n)\right\} \mathbb{P}\left\{X_{n+1}-X_{n}>h(n)\right\}\right. \\
& \left.+\int_{-\infty}^{-h(n)} \mathbb{P}\left\{X_{n} \in \mathrm{d} y\right\} \mathbb{P}\left\{X_{n+1}-X_{n} \geq-y\right\}\right) \\
= & \frac{\mathbb{P}\left\{X_{n+1} \geq 0, X_{n+1}-X_{n} \geq h(n)\right\}}{\mathbb{P}\left\{X_{1-\varepsilon} \geq 0\right\}} .
\end{aligned}
$$

After applying (A.12), it is clear that $P_{2}=o\left(\mathbb{P}\left\{X_{n}>0\right\}\right)$. Finally,

$$
\begin{aligned}
P_{3} & \leq \mathbb{P}\left\{X_{n} \geq h(n), X_{n+\varepsilon}-X_{n}<-h(n)\right\} \\
& \leq \mathbb{P}\left\{X_{n} \geq 0\right\} \mathbb{P}\left\{X_{\varepsilon}<-h(n)\right\} \\
& =o\left(\mathbb{P}\left\{X_{n} \geq 0\right\}\right) .
\end{aligned}
$$

Now we should make use of the fact that if (A.10) holds for any fixed $\varepsilon \in(0,1)$ then it holds uniformly in $\varepsilon \in(0,1)$. Consequently, (2.1) holds for $a(t)=\mathbb{P}\left\{X_{t} \geq 0\right\} / t$. The proof of (A.9) is similar. Finally, condition (2.2) for the function $a(t)$ follows from the dominated convergence theorem and the fact that

$$
\int_{1}^{y-1} \frac{a(y-t) a(t)}{a(t)} \mathrm{d} t \leq C \sum_{k=1}^{[y-1]} \frac{a(k) a([y-1]-k)}{a[y-1]}<\infty
$$

for some constant $C$. This completes the proof of Lemma A.2.

\section{A.2. Proof of Theorem 2.2}

For Lévy processes, the result follows directly from Theorem 2.1 and Lemma A.2. For random walks, it can be proved along the lines of [13]. The only difference is that we should apply our Lemma A.1 instead of Lemma 4 of [13].

\section{Appendix B. Lévy processes}

In this section we collect some facts from the theory of Lévy processes that we use in this paper.

The following lemma and its proof may be found in [29, Lemma 30.3].

Lemma B.1. Let $X_{t}$ be a Lévy process on $\mathbb{R}^{d}$. For any $\varepsilon>0$, there exists $C=C(\varepsilon)$ such that, for any $t$,

$$
\mathbb{P}\left\{\left|X_{t}\right|>\varepsilon\right\} \leq C t .
$$

There exist $C_{1}, C_{2}$, and $C_{3}$ such that

$$
\mathbb{E}\left\{\left|X_{t}\right|^{2} ;\left|X_{t}\right| \leq 1\right\} \leq C_{1} t, \quad\left|\mathbb{E}\left\{X_{t} ;\left|X_{t}\right| \leq 1\right\}\right| \leq C_{2} t, \quad \mathbb{E}\left\{\left|X_{t}\right| ;\left|X_{t}\right| \leq 1\right\} \leq C_{3} t^{1 / 2},
$$

for any $t$. 
The next theorem is a version of [29, Theorem 25.3] adapted to our needs.

Theorem B.1. Let $X_{t}$ be a Lévy process on $\mathbb{R}^{d}$ with the Lévy measure $v$. Then $X_{t}$ has a finite exponential moment if and only if $[v]_{|x|>1}$ has a finite exponential moment.

Let $F$ be an infinitely divisible law on $[0,+\infty)$. Its Laplace law can be expressed as (see [29, Theorem 30.1])

$$
\left.\int_{0}^{\infty} \mathrm{e}^{-\lambda x} F(\mathrm{~d} x)=\exp \left\{-\gamma \lambda-\int_{0}^{\infty}\left(1-\mathrm{e}^{-\lambda x}\right) \nu(\mathrm{d} x)\right)\right\},
$$

where $\gamma \geq 0$ is a constant and $v$ is a Borel measure on $(0, \infty)$ for which $\mu \equiv v(1, \infty)<\infty$ and $\int_{0}^{1} x v(\mathrm{~d} x)<\infty$.

Theorem B.2. ([16, Theorem 1].) For $F$ infinitely divisible on $[0,+\infty)$, the following assertions are equivalent:

(i) $F \in 8$;

(ii) $\mu^{-1} v(1, x] \in \delta$;

(iii) $\bar{F}(x) \sim \bar{v}(x)$.

We also need a density version of this theorem.

Theorem B.3. Let the infinitely divisible law $F$ have a density $f$. Assume that there exists $x_{0}$ such that $v$ has a density $g(x)$ for $x>x_{0}$. If $g(x)$ belongs to the class $\delta d(\gamma)$ then

$$
\lim _{x \rightarrow \infty} \frac{f(x)}{g(x)}=\int_{0}^{\infty} \mathrm{e}^{\gamma x} f(x) \mathrm{d} x .
$$

We can prove this theorem exactly as [23, Theorem 3.1] for a distribution function from $\delta(\gamma)$. Corresponding properties of the class $\$ d(\gamma)$ may be found in [20, Section 3].

\section{Acknowledgements}

We would like to thank Serguei Foss for drawing attention to this problem and a number of helpful discussions. We are also grateful to Onno Boxma, Ton Dieker, and Bert Zwart for many useful comments and suggestions that helped to improve the manuscript. Part of this work was done while Vsevolod Shneer was visiting EURANDOM. He would like to thank EURANDOM for its hospitality.

\section{References}

[1] Авate, J. And Whitt, W. (1997). Asymptotics for M/GI/1 low-priority waiting-time tail probabilities. Queueing Systems 25, 173-233.

[2] Asmussen, S., Klüppelberg, C. and Sigman, K. (1999). Sampling at sub-exponential times, with queueing applications. Stoch. Process. Appl. 79, 265-286.

[3] Baltrūnas, A., Daley, D. J. And Klüppelberg, C. (2004). Tail behaviour of the busy period of a $G I / G I / 1$ queue with subexponential service times. Stoch. Process. Appl. 111, 237-258.

[4] Bertoin, J. (1996). Lévy Processes. Cambridge University Press.

[5] Bertoin, J. and Doney, R. A. (1996). Some asymptotic results for transient random walks. Adv. Appl. Prob. 28, 207-226.

[6] Bingham, N. H., Goldie, C. M. And Teugels, J. L. (1987). Regular Variation. Cambridge University Press.

[7] Borovkov, A. A. (1962). New limit theorems in boundary problems for sums of independent terms. Siberian Math. J. 3, 645-694. 
[8] Borovkov, A. A. (2004). On the asymptotics of distributions of first-passage times. I. Math. Notes 75, $23-37$.

[9] Borovkov, A. A. (2004). On the asymptotics of distributions of first-passage times. II. Math. Notes 75, 322-330.

[10] Cohen, J. W. (1982). The Single Server Queue, 2nd edn. North-Holland, Amsterdam.

[11] De Meyer, A. And Teugels, J. L. (1980). On the asymptotic behaviour of the distributions of the busy period and service time in $M / G / 1$. J. Appl. Prob. 17, 802-813.

[12] Denisov, D., Dieker, A. B. And Shneer, V. (2008). Large deviations for random walks under subexponentiality: the big-jump domain. Ann. Prob. 36, 1946-1991.

[13] Doney, R. A. (1989). On the asymptotic behaviour of first passage times for transient random walk. Probab. Theory Relat. Fields 81, 239-246.

[14] Doney, R. A. And Maller, R. A. (2004). Moments of passage times for Lévy processes. Ann. Inst. H. Poincaré Prob. Statist. 40, 279-297.

[15] Embrechts, P. AND Hawkes, J. (1982). A limit theorem for the tails of discrete infinitely divisible laws with applications to fluctuation theory. J. Austral. Math. Soc. Ser. A 32, 412-422.

[16] Embrechts, P., Goldie, C. M. and Veraverbeke, N. (1979). Subexponentiality and infinite divisibility. Z. Wahrscheinlichkeitsth 49, 335-347.

[17] Foss, S. AND Korshunov, D. (2000). Sampling at a random time with a heavy-tailed distribution. Markov Process. Relat. Fields 6, 543-568.

[18] Jelenković, P. R. AND MomČIlović, P. (2004). Large deviations of square root insensitive random sums. Math. Operat. Res. 29, 398-406.

[19] KlüPPelberg, C. (1988). Subexponential distributions and integrated tails. J. Appl. Prob. 25, 132-141.

[20] Klüppelberg, C. (1989). Subexponential distributions and characterizations of related classes. Prob. Theory Relat. Fields 82, 259-269.

[21] Kyprianou, A. E. And Palmowski, Z. (2006). Quasi-stationary distributions for Lévy processes. Bernoulli 12, 571-581.

[22] Mogul'skIĬ, A. A. (2006). Large deviations of the first passage time for a random walk with semiexponentially distributed jumps. Siberian Math. J. 47, 1084-1101.

[23] Pakes, A. G. (2004). Convolution equivalence and infinite divisibility. J. Appl. Prob. 41, 407-424.

[24] Palmowski, Z. And Rolski, T. (2006). On the exact asymptotics of the busy period in GI/G/1 queues. Adv. Appl. Prob. 38, 792-803.

[25] Petrov, V. V. (1965). On the probabilities of large deviations for sums of independent random variables. Theory Prob. Appl. 10, 287-298.

[26] Petrov, V. V. (1975). Sums of Independent Random Variables. Springer, New York.

[27] Rogozin, B. A. (1966). Distribution of certain functionals related to boundary value problems for processes with independent increments. Theory Prob. Appl. 11, 656-670.

[28] RozovskIĬ, L. V. (1993). Probabilities of large deviations on the whole axis. Theory Prob. Appl. 38, 53-79.

[29] Sato, K.-I. (1999). Lévy Processes and Infinitely Divisible Distributions. Cambridge University Press.

[30] Zwart, A. P. (2001). Tail asymptotics for the busy period in the GI/G/1 queue. Math. Operat. Res. 26, $485-493$. 